\title{
ISCHIOFEMORAL IMPINGEMENT - AN ETIOLOGY OF HIP PAIN: CASE REPORT
}

Carlos Massao Aramaki Yanagishita', Guilherme Guadagnini Falótico', Davi Araújo Veiga Rosário², Gustavo Gambuggi Pugina³, André Azambuja Neves Wever ${ }^{4}$, Edmilson Takehiro Takata ${ }^{5}$

\section{ABSTRACT}

An association between hip pain and quadratus femoris muscle abnormality on magnetic resonance imaging (MRI) with concurrent narrowing of the ischiofemoral space has been reported in the recent literature. This raises the possibility that the muscle damage observed is due to ischiofemoral impingement. This diagnosis has been noted in middle-aged females either with or without a history of trauma or surgery, is a rarely described feature. We report here on a 31-year-old woman who presented with non-traumatic hip pain and evidence of narrowing of the ischiofemoral space and edema in the quadratus femoris. Nonsurgical treatment was administered, which relieved her hip pain. The diagnosis of ischiofemoral impingement should be considered in female patients complaining of hip pain without any other evident cause.

Keywords - Hip; Sciatic Nerve; Ischium; Femur

\section{INTRODUCTION}

The growing number of magnetic resonance imaging (MRI) exams in the investigation of hip pain, as well as a better interpretation of these tests, allow for the verification of less common changes that mimic joint or extra-articular diseases of the hip.

The association between hip pain and signal changes in the quadratus femoris muscle (QFM) on MRI has been discussed in the current literature. The decrease of the space between the ischial tuberosity and the lesser trochanter, together with consequent ischiofemoral impingement during the repeated movement of the hip, may be responsible for injury to the quadratus femoris muscle ${ }^{(1)}$. The initial description of ischiofemoral impingement in two patients after hip arthroplasty and in one patient after osteotomy of the proximal femur was published in the 1970s and attributed the impingement to an increase in the size of the lesser trochanter and ischiofemoral narrowing ${ }^{(2)}$.

Recently, the reduction of the ischiofemoral space was also observed in patients without previous hip surgery and those without any history of trauma ${ }^{(1-3)}$, as well as quadratus femoris muscle signal changes on MRI also being observed in patients without ischiofemoral space narrowing ${ }^{(4-6)}$, keeping the pathogenesis of ischiofemoral impingement uncertain.

This case report aims to demonstrate the possible relationship between ischiofemoral impingement and quadratus femoris muscle changes in MRI scans, as well as raising the possibility of diagnosing ischiofemoral impingement in cases of hip pain with no obvious cause.

\section{CASE REPORT}

Female patient, 31 years old, with a history of pain in the posterior region of the right hip for four years, with no history of trauma, with insidious onset and progressive worsening, especially on exertion. She sought care from other orthopedic services, was diagnosed as and treated clinically for mechanical low back pain. As there was no improvement, she sought the Adult Hip Group eight months ago at the

\footnotetext{
1 - Medical Intern, Hip Group, Paulista School of Medicine - Unifesp - São Paulo, SP, Brazil.

2 - Resident, Department of Orthopedics and Traumatology, Paulista School of Medicine - Unifesp - São Paulo, SP, Brazil.

3 - Medical Intern, Hip Group, Hospital IFOR - São Bernardo do Campo, SP, Brazil.

4 - Assistant Physician, Hip Group, Paulista School of Medicine - Unifesp - São Paulo, SP, Brazil.

5 - Head, Hip Group, Paulista School of Medicine - Unifesp; Master's degree in Orthopedics by the Paulista School of Medicine - Unifesp - São Paulo, SP, Brazil.

Study conducted at the Department of Orthopedics and Traumatology, Paulista School of Medicine - Unifesp.

Correspondence: Rua Borges Lagoa, 1.065, conjunto 126 - Vila Clementino - 04038-030 - São Paulo, SP, Brazil. Email: cmassao5@yahoo.com.br

Received for publication: 10/25/2011, accepted for publication: 12/21/2011.
}

The authors declare that there was no conflict of interest in conducting this work 
Paulista School of Medicine/Unifesp. At the time, she reported deep gluteal pain on the right, without irradiation, not related to the position of the body. On physical examination, she presented normal muscle trophism and antalgic gait on the right side. The range of motion of the hips was normal. Among the special maneuvers, there was a positive Freiberg test ${ }^{(7)}$ and pain when performing the maneuver in the lateral decubitus position with the hip in extension, adduction, and internal and external rotation movements (test suggested by the authors). The Lasègue, FADURI (flexion, adduction, and internal rotation), piriformis test, and Pace sign were negative.

Additional tests included radiographic examinations of the pelvis and right hip demonstrating a valgus femoral neck, bilateral ischiofemoral space narrowing, and the presence of cysts in the right ischium (Figure 1). Faced with clinical and radiographic findings and diagnostic uncertainty, we requested a pelvic MRI, which showed increased signal in the quadratus femoris muscle on T2-weighted sequences (Figure 2). Once the diagnosis of ischiofemoral impingement was made, non-surgical treatment was introduced, based on a non-hormonal antiinflammatory drug for seven days and daily physical therapy for stretching and strengthening the pelvic muscles, with progressive improvement of symptoms. After three months of treatment, the patient showed significant functional improvement, and resumed Pilates activities without any restriction.

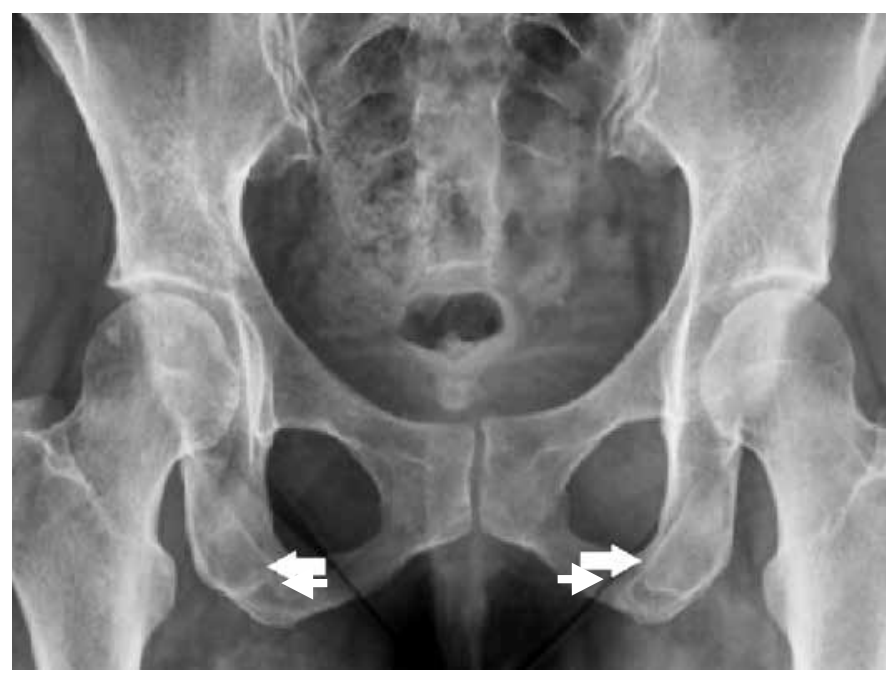

Figure 1 - AP radiograph of the pelvis showing valgus femoral neck, ischial cysts (arrows) and a close relationship between the lesser trochanter and ischium.

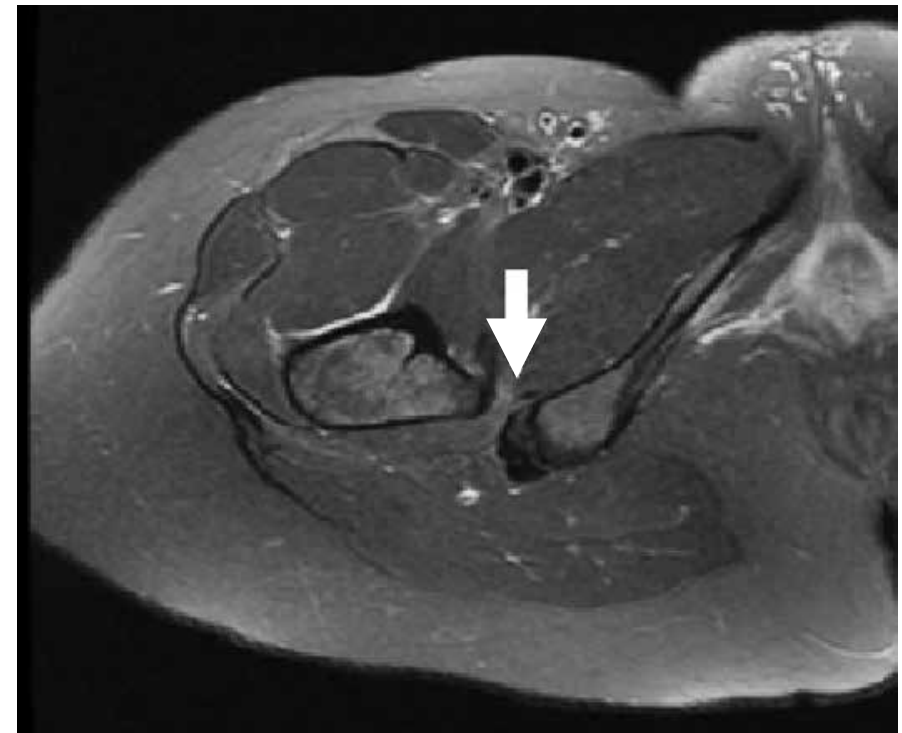

Figure 2 - Female patient, 31 years - Axial T2 MRI demonstrating ischiofemoral space narrowing and hypersignal on the quadratus femoris muscle.

\section{DISCUSSION}

Our objective with the above report is to demonstrate to orthopedists this new entity, which tends to be more commonly diagnosed with the expansion in the use of MRI in the investigation of hip pain. We will cover the anatomy, clinical and radiological aspects, and possible treatments of ischiofemoral impingement.

\section{Anatomy}

The quadratus femoris muscle (QFM) is located on the posterior aspect of the hip joint (Figures 3 and 4). It originates in the inferolateral margin of the ischium, above the ischial tuberosity, immediately prior to the hamstring muscles. The insertion occurs along the posteromedial portion of the proximal femur, in the quadrate tubercle on the posterior intertrochanteric line. The fibers of the QFM have a horizontal orientation and are interspersed with fat in its ischial portion ${ }^{(8)}$.

The anatomical relations are: anteriorly with the external obturator muscle and posteriorly with the sciatic nerve. On its superior aspect is the inferior gemellus muscle and the adductor magnus is at its inferior margin ${ }^{(8)}$ (Figure 4).

\section{Innervation and function}

The quadratus femoris muscle is innervated by a small branch of the sacral plexus, formed by the roots of $\mathrm{L} 4, \mathrm{~L} 5$ and $\mathrm{S} 1$. The nerve to the QFM 


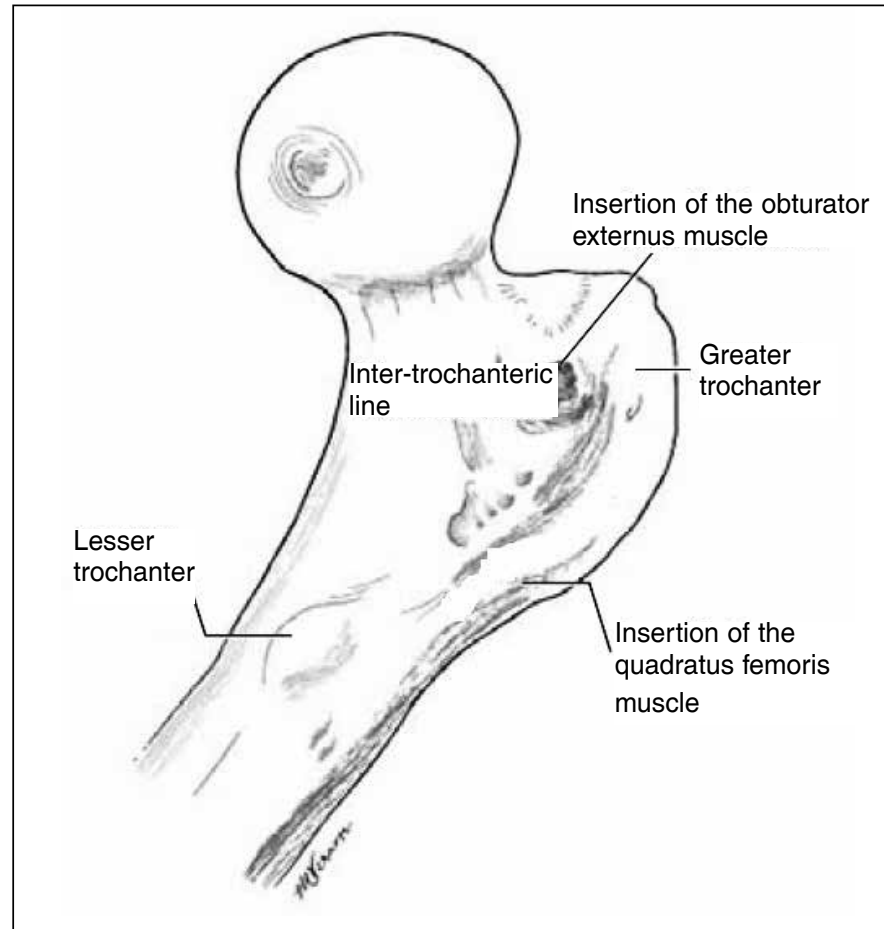

Figure 3 - Posterior view of the proximal femur with muscle insertions.

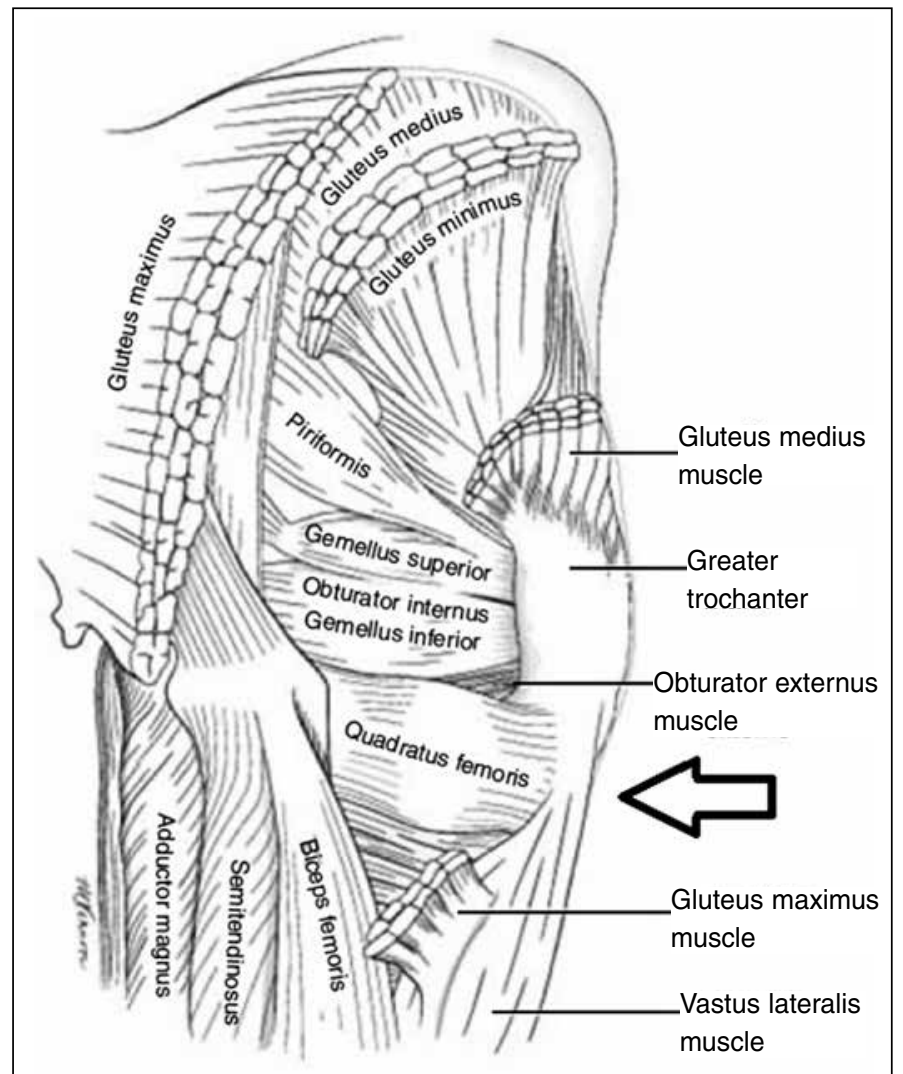

Figure 4 - Posterior view of the hip, intermuscular relationships. Quadratus femoris muscle (arrow).

externalizes at the pelvis through the greater sciatic notch, along an anteroinferior path to the gemellus muscles and obturator internus, and penetrates the
QFM on its anterior surface. This muscle performs adduction and external hip rotation ${ }^{(8)}$.

\section{Clinical evaluation of patients}

Clinical assessment of patients with ischiofemoral impingement is quite difficult since the complaints are vague and imprecise and may be confused with other lumbar diseases, intra- or extra-articular hip diseases, including deep gluteal pain. In our case, we observed an exacerbation of symptoms through the maneuver performed in the lateral decubitus position with hip extension, adduction, and internal and external rotational movements (test suggested by the authors), as well as the Freiberg test ${ }^{(7)}$, which is used in piriformis syndrome research and consists of pain upon internal rotation with the hip in extension.

\section{Evaluation of MR images}

Obviously, the various sections of MRI are complementary and necessary, but the QFM is better evidenced in axial sections $^{(4,8)}$ (Figure 2). In this projection, it is possible to assess its origin, insertion, as well as their anterior and posterior relationships. The sciatic nerve and hamstring muscles are posteriorly related and must be carefully inspected because they are possible sites of injury. There is a convergence of fibers at the femoral insertion ${ }^{(8)}$.

\section{Types of injuries to the quadratus femoris muscle}

Lesions of the QFM are described as myotendinous strain, partial rupture, and impingement on the muscle belly ${ }^{(4,5)}$.

Impingement injuries are more common in women of middle age and older, with posterior gluteal pain or increasing pain with or without a considerable initial traumatic event ${ }^{(8)}$. The pain may radiate to the back of the thigh, possibly by irritation of the sciatic nerve which is on the posterior portion of the muscle $e^{(9,10)}$.

Myotendinous strain presents with muscle edema with or without the presence of liquid, seen mostly in its femoral portion, while the ruptures can be evidenced by edema and liquid posterior to the lesser trochanter $^{(8)}$.

\section{Ischiofemoral impingement}

In 1977, Johnson ${ }^{(2)}$, and later Patti et al ${ }^{(3)}$, associated the ischiofemoral narrowing with hip pain. Kassarjian $^{(5)}$ reports impingement between the space 
of the ischium or hamstrings and the posteromedial portion of the femur, compressing the quadratus femoris muscle. An anatomical variation in the origin of the hamstring muscles could also cause soft tissue impingement without significant ischiofemoral narrowing ${ }^{(1)}$.

Several etiologies have been suggested: increased femoral diameter at the level of the lesser trochanter, a congenital posteromedial femoral position, reduction of the horizontal offset in hip arthroplasty, valgus femoral neck, postoperative period of valgus osteotomy, malunion of intertrochanteric fractures, coxarthrosis with superomedial migration, and hip osteochondromas $^{(1-3)}$. Female morphology, with a broad, shallow pelvis, is predisposed to ischiofemoral impingement, and all of the cases documented in the literature are in women ${ }^{(1)}$.

Radiographic findings are infrequent and, when present, are nonspecific for ischiofemoral impingement. Patti et $\mathrm{al}^{(3)}$ reported the presence of heterogeneous sclerosis in the lesser trochanter and in the ischium in one of their cases.

In the MRI, signal changes for this injury are typically centered on the muscle belly, the site of maximum compression (Figure 2), different from the edema caused by myotendinous strain that occurs to the distal myotendinous junction ${ }^{(8)}$. In their series of 12 hips, Torriani et al ${ }^{(1)}$ observed edema in all cases, partial tears in 33\% and fatty infiltration in $8 \%$.

Other entities involving the QFM, such as tendinopathy $^{(9)}$ and degenerative lesions of the sacral branches innervating the quadratus femoris muscle, may be the cause of hip pain. Finding fluid collection in the region of the lesser trochanter should be differentiated from bursitis of the iliopsoas muscle, while collections near the ischial tuberosity have ischial bursitis as a differential diagnosis ${ }^{(8)}$.

Some authors, like Torriani et $\mathrm{al}^{(1)}$, have attempted to correlate a narrowing between the lesser trochanter and the ischial tuberosity with the QFM impingement. However, due to the small sample of cases and difficulty in controlling the rotation of the limb at the time of imaging, this correlation has uncertain validity.

There is great difficulty in formulating a diagnosis of this entity, since the history and physical examination are imprecise and difficult to interpret ${ }^{(9-11)}$. Therefore, MRI is essential for correct diagnosis ${ }^{(4)}$.

Among the possible treatments that have been described are appropriate stretching exercises ${ }^{(10)}$, infiltrations with methylprednisolone acetate ${ }^{(11)}$, neurostimulation and percutaneous ultrasound therapy ${ }^{(9)}$. As was true in our case, O'Brien and Bui-Mansfield ${ }^{(4)}$, in their study, found that non-surgical interventions were adequate for a good resolution in cases of hip pain due to inflammation of the quadratus femoris muscle.

The ischiofemoral impingement represents one of the causes of hip pain. Although uncommon, it should be recognized and adequately addressed by orthopedists, because only with a larger series and by sharing experiences can a standard be established for clinical diagnosis and uniform treatment of this entity.

\section{REFERENCES}

1. Torriani M, Souto SC, Thomas BJ, Ouellette H, Bredella MA. Ischiofemoral impingement syndrome: an entity with hip pain and abnormalities of the quadratus femoris muscle. AJR Am J Roentgenol. 2009;193(1):186-90.

2. Johnson KA. Impingement of the lesser trochanter on the ischial ramus after total hip arthroplasty. Report of three cases. J Bone Joint Surg Am. 1977;59(2):268-9.

3. Patti JW, Ouellette $\mathrm{H}$, Bredella MA, Torriani M. Impingement of lesser trochanter on ischium as a potential cause for hip pain. Skeletal Radiol. 2008;37(10):939-41.

4. O'Brien SD, Bui-Mansfield LT. MRI of quadratus femoris muscle tear: another cause of hip pain. AJR Am J Roentgenol. 2007;189(5):1185-9.

5. Kassarjian A. Signal abnormalities in the quadratus femoris muscle: tear or impingement? AJR Am J Roentgenol. 2008;190(6):W379.

6. Bui-Mansfield LT, O'Brien SD. Reply to letter. Am J Roentgenol. 2008;190(6):W380-1.
7. Freiberg $A H$, Vinke TH. Sciatica and the sacroiliac joint. J Bone Joint Surg Am 1934;16:126-36.

8. Kassarjian A, Tomas X, Cerezal L, Canga A, Llopis E. MRI of the quadratus femoris muscle: anatomic considerations and pathologic lesions. AJR Am J Roentgenol. 2011;197(1):170-4.

9. Peltola K, Heinonen OJ, Orava S, Mattila K. Quadratus femoris muscle tear: an uncommon cause for radiating gluteal pain. Clin J Sport Med. 1999;9(4):228-30.

10. Willick SE, Lazarus M, Press JM. Quadratus femoris strain. Clin J Sport Med. 2002;12(2):130-1.

11. Klinkert $P$ Jr, Porte RJ, de Rooij TP, de Vries AC. Quadratus femoris tendinitis as a cause of groin pain. $\mathrm{Br} J$ Sports Med. 1997;31(4):348-9. 